\title{
Multi-role collaborative fire drill simulation with smoke risk evaluation
}

\author{
Chen $\operatorname{Lin}^{1, a}$, Xiaobin $\operatorname{Lin}^{1, b, *}$ \\ ${ }^{1}$ Department of Physics and Electronic Information Engineering, MinJiang University, Fuzhou \\ 350108, China \\ alinchen1988@foxmail.com, ${ }^{\text {b3 } 33004797 @ q q . c o m ~}$
}

Keywords: Virtual Reality, VIZARD, KBENGINE, fire dynamics data, smoke risk evaluation model.

\begin{abstract}
Fire is one of the major disasters that often happen in the cities. It causes serious economic losses and casualties. Smoke is the main cause of death in fire and it is difficult to be simulated in the traditional way of fire drill. A multi-role collaborative fire drill simulation system was developed on the basis of virtual reality and server engine technology. The system consists of the client end and the server end. In the client end, the realization of the virtual fire scenes is based on the VIZARD software, which allows the trainees to experience a realistic and yet non-threatening fire scenes. The KBENGINE framework is used to enable multi-role synchronous login, the participants can collaborate in a virtual fire drill without time or space constraints. In order to carry out effective virtual training, Fire dynamics data and three-dimensional rendering technology has been especially designed to create a realistic and accurate smoke environment. The smoke risk evaluation model was introduced to assess the safety of different evacuation routes so that trainees could identify the safest route to escape. The test results show that the system has good performance and high accuracy, which helps trainees learn the knowledge of firefighting in the low-cost, high-security, high-fidelity and repeatable virtual environment, and accumulate the real-world firefighting combat experience.
\end{abstract}

\section{Introduction}

Fire is one of the major disasters that often lead to serious economic losses and deaths in the cities[1]. How to safely escape from the fire site has become the focus of fire drills. According to an American report [2], the number of deaths due to smoke in the fires is as high as two-thirds of the total deaths. In many fire accidents, the hazard caused by smoke seriously hinders trapped people from looking for a safe evacuation or rescue route. Therefore, reducing the impact of smoke hazards is a key issue for effectively reducing fire casualties. Fire information and drill are the common ways to learn about fire prevention at present. The former popularizes fire knowledge theoretically and the latter introduces the practical experience in fire protection. However, it may generate high social and economic costs and even human casualties due to the potential risks, such as toxic gases and uncontrolled fires, etc [3].

Contrary to the traditional fire dill with potential risk, a low-cost, high-security, highly-simulated, repeatable Virtual Reality (VR) drill system allows trainees to simulate evacuation or rescue training in virtual scenarios. A number of VR fire training systems have been proposed by different researchers, for example, a VR training simulator integrated fire dynamic data was developed by Cha et al [4]. A fire evacuation simulation method based on numerical simulation in VR environment was proposed by Ren et al [5]. A VR simulator with smoke hazard assessment capacity was proposed by $\mathrm{Xu} \mathrm{Z}$. et al [6] and applied it to two simple stand-alone scenarios. However, the virtual fire drill system that combines smoke risk evaluation model and the real fire scenario with multi-person and multi-role collaboration has not been further studied. Therefore a smoke risk evaluation model is proposed in this research. The multi-role simulation engine can not only identify the safest evacuation or rescue path based on fire and smoke hazard, but also simulate joint fire drill without geographic restriction.

Three technologies were adopted in the development of the multi-role synchronous simulation fire training system: (1) The system is based on client/server architecture. In the client end, Vizard was employed to design the virtual scenery for fire drill simulations. The KBENGINE framework was 
used in the server end for simultaneous multi-role login so as to realize collaborative virtual fire drill without the constraints of time and location. (2) In the research the fire dynamics data was simulated in combination with three-dimensional rendering technology to generate a real and accurate virtual fire smoke environment. Smoke impairs the vision of trapped people and firefighters in evacuation or rescue, which makes it hard to identify exits and hence impedes evaluation [7]. Therefore in the virtual fire drill, accurate smoke simulation is crucial to the success of an effective evacuation or rescue. Some simple methods have been used by researchers to simulate the dynamic evolution of smoke [8-10], such as cellular automaton, particle systems and random variables. In order to achieve realistic smoke simulation, it is necessary to combine the important characteristics of smoke, which are its distribution and density. Several Computational Fluid Dynamics (CFD) programs [11] can output smoke data with high accuracy. The Fire Dynamics Simulator (FDS) developed by the National Institute of Standards and Technology (NIST) has an error range of 5-20\% from the actual value and is widely used in various fire researches [12]. With the assistance of 3D rendering technology, effective and accurate simulation of fire smoke can be achieved. (3) The smoke risk evaluation model is introduced in this research to assess smoke hazard from the evacuation or rescue routes to help trainees find the safest route. The key direct hazards of smoke are toxicity and heat. Obviously, both toxicity and heat have a serious impact on the safety of possible evacuation or rescue routes. Fractional Effective Dose (FED) was used to evaluate the risk of smoke [13]. In addition, some studies of thermal effects have identified the highest smoke temperature human beings can tolerate in fires [14-17], and the 6-Gas model [18] based on FED was proposed by NIST has been used to describe the evaluation of smoke heat hazard [15]. The test results show that the system has good performance and is good for both trapped people and firefighters.

\section{Multi-role collaborative fire drill simulation with smoke risk evaluation}

The system, supported by the fire dynamics data of FDS and 3D rendering technology, adopts VIZARD as VR platform and KBENGINE as server framework. Smoke risk evaluation model was introduced to evaluate the safety of different evacuation or rescue routes, so that trainees can identify the safest escape route. The flowchart of the proposed system for fire drill training was shown in Fig. 1 .

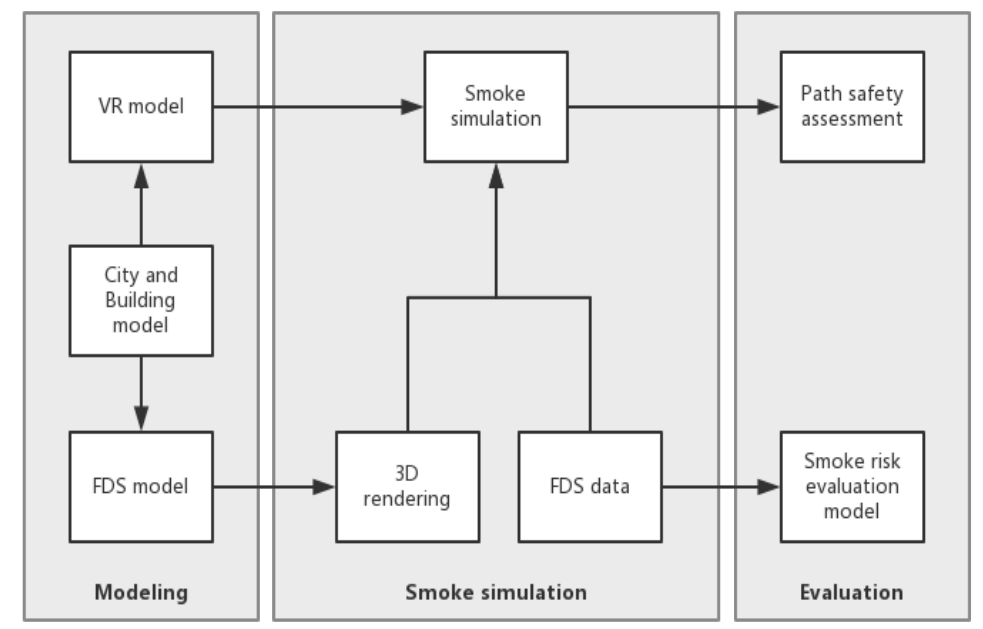

Fig. 1 The flowchart of the proposed system for fire drill training

\section{1 multi-role synchronous simulation fire drill system}

The client end of this multi-role synchronous simulation fire drill system consists of virtual city scene module, small map function module, flame simulation module, role control and rescue module. The virtual city scene module uses 3DSMAX to construct the prefabricated model of the virtual city scene, for example, the high building, the road, the sidewalk, and then these models were imported into the VIZARD using the OSGB format to reproduce the virtual city scene. Finally, the scene is 
optimized through ambient light and color adjustment to improve visual reality. Tools in Vizard such as cameras, UI rendering elements, and shading masks were used to visualize the real-time dynamics of fire and people and the small map. The flame of the virtual fire scene was simulated by particle system. The control of different roles such as fireman, police and medical staff was carried out via avatar, collision mechanism and interaction function in VIZARD. Different roles were assigned with different skills. Three different difficulty levels were designed, among which the elements such as ignition points and personnel configuration are fixed, as shown in Table 1. Customized mode was also designed to allow dynamical configuration of important elements in the fire drill simulation. Trainees can freely configure the elements in virtual fire drill for a better training effect. Until now, a stand-alone version of the simulated fire drill system has been implemented.

Table 1 the settings of fire drill elements under different difficulty levels

\begin{tabular}{ccccccc}
\hline $\begin{array}{c}\text { Element } \\
\text { Mode }\end{array}$ & $\begin{array}{c}\text { Ignition } \\
\text { Points }\end{array}$ & $\begin{array}{c}\text { Firema } \\
\text { n }\end{array}$ & $\begin{array}{c}\text { Trapped } \\
\text { Staff }\end{array}$ & $\begin{array}{c}\text { Crow } \\
\text { d }\end{array}$ & Police & $\begin{array}{c}\text { Medical Assistance } \\
\text { Department }\end{array}$ \\
\hline Simple & 1 & 3 & 1 & 1 & 1 & 1 \\
Medium & 3 & 6 & 5 & 10 & 3 & 3 \\
$\begin{array}{c}\text { difficult } \\
\mathbf{y}\end{array}$ & 5 & 10 & 10 & 15 & 5 & 3 \\
\hline
\end{tabular}

The multi-role simultaneous simulation of virtual fire drill, with the data exchange functionality supported by a server, allows more than one simultaneous login into the same scene for collaborative training. Therefore, a login interface was designed for the client end. The Vizard command "viz.addChild" was invoked to load the scene as the login interface background, and the corresponding functions were realized via Vizard build-in GUI controls and PYTHON scripts. In summary, the functions required by the cliend end of the simulation system are completed.

The server end of the simulation system consists of four modules: server framework construction, server AI role control, data exchange, and login interface interaction. The server environment in this research was developed based on the opensource server engine KBENGINE, which supports multi-client synchronization load, easy real-time data exchange between server and clients, data synchronization of each client, a variety of plug-ins for the integration of other technologies to form a complete system. The server-side framework build is mainly concerned with software installation and parameter settings, i.e. the installation of PYTHON parser and MYSQL database. The command "[mysqld]" and "lower_case_table_names = 0 " were set in the my.ini file to enable case sensitivity in MYSQL database. A KBE account was created and set to disable null password logins. After the above configurations, the"! (Win) startdemo_server_orge.bat" and the "! (Win) startdemo_client_orge.bat" were run to start the server end and the client end. The server-side role AI was implemented by calling "call SetDefendPlayer", "call PlayGame" and "call StartThread" scripts. The role data controlled by the server-side AI was synchronized to each client in real time, and the client was displayed in real time after receiving the server-side role data, which enables more vivid and effective simulation of real-world people. In this research, AI scripts were used to control the on-site spectators in the virtual fire scene to move toward the fire point. During the movement, collision detection was performed to force retreat once the AI-controlled spectators had moved into a dangerous distance of the flame. The data exchange was realized through entity. The entity.xml file on the server creates an entity, configurates parameters relating to entity such as Client, Cell, and Base, and guarantees the match of the files defined by the client and the server. Login interaction involves the client end sending a login request with specific computer address and port information, and then the server queries DBMgr to verify the user name and password. Meanwhile the server forwards the request to BaseappMgr, BaseappMgr sends a message to the Baseapp with the minimal load, then Baseapp creates a Proxy whose port return to the client via BaseappMgr, DBMgr, Loginapp to complete the login function. Compared to the login function, the difference of the registration function is that it writes user name and password into the database during data exchange. 
The client end, together with the server side, constitutes a complete multi-role collaborative virtual fire drill system.

\section{2 fire smoke simulation based on FDS}

FDS data and 3D rendering technology were used to realistically and effectively simulate the smoke in the actual fire scenes. FDS data has been verified to be able to provide accurate fire dynamic results [19], and 3D rendering technology has also been verified to be very suitable for integration with FDS grid data [20]. Therefore the combination of FDS data and 3D rendering technology makes a perfect tool for simulating accurate and realistic smoke.

FDS uses grid systems to divide the space into small volumes of specific thermal properties [12]. In FDS, the continuous time is divided into several time segments, and the smoke density of each grid is obtained in any time segment [12]. In smoke simulation, a 3D grid voxel that is volume element used in 3D rendering [20] and is created in the VR platform following FDS grid. The opacity value of a voxel can be obtained by the normalized soot density values in the corresponding grid, which is used to accurately represent the low visibility caused by smoke. The normalized formula is shown in eq. (1).

$$
\alpha=\frac{D_{s}}{D_{\operatorname{minax}}}
$$

In the above equation, ${ }^{\alpha}$ represents the normalized soot density value in the range of 0 to 1 , the larger the value, the more opaque the lower the visibility. $D_{s}$ and $D_{s \max }$ respectively represents the value of the soot density and the maximum of the soot density of the corresponding grid. During 3D rendering, the opaque voxel grid values for each time segment are dynamically adjusted based on the ash density values. Using this approach, accurate dynamic soot evolution simulations can be truly visualized in a multi-role synchronous simulation fire drill system.

\section{3 smoke risk evaluation model}

Some researchers found that the main hazards of smoke include toxicity and heat [14-17]. The smoke toxicity is the result of a combination of gases and FED is an important toxic model during the 30-minute exposure period [13]. The comprehensive toxicity (ie, CO, CO2, $\mathrm{HCN}, \mathrm{O} 2, \mathrm{HCl}$, and $\mathrm{HBr}$ ) of the six gases commonly found in smoke is assessed using the 6-Gas model [18] based on the FED given in eq. (2).

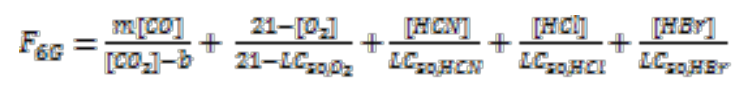

$\left[C_{i}\right]$ in square brackets indicates the mean atmospheric concentration $[(\% \times \min ) / \mathrm{min}]$ during the 30-minute exposure period. The formula is as shown in (3).

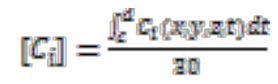

Where $c_{\mathrm{i}}\left(x_{s} y_{s} z_{s} t\right)$ represents the atmospheric concentration function of the ith gas (ie CO, CO2, HCN, $\mathrm{O} 2, \mathrm{HCl}$ and $\mathrm{HBr}$ ). The variables $\mathrm{c}$ and $\mathrm{d}$ represent the initial point and the end point of the route, respectively. The atmospheric concentrations of these gases are obtained by FDS and the results are accurate within $20 \%$ of the experimental measurements $[11,12,19]$. The variables $\mathrm{m}$ and $\mathrm{b}$ are used to indicate the combined toxic effects of $\mathrm{CO}$ and $\mathrm{CO} 2$. After 30 minutes of exposure, LC_50 is a toxic gas that causes $50 \%$ of laboratory animals to die [21]. The higher $F_{6 G}$ is, the stronger the comprehensive toxicity of smoke. When $F_{6 G}$ attains 1 , there is a high mortality rate of smoke toxicity $[13,21]$.

The thermal hazards include convection and radiation. During the exposure period, the fractional dose based on FED can be obtained by calculating the sum of radiation and convection fraction, as expressed in (4) [15]:

$$
F_{t}=\sum_{t_{2}}^{t_{2}}\left(\frac{1}{t_{p}}+\frac{1}{t_{2}}\right) \Delta t
$$

Where $\Delta t$ is a fixed time interval between time segments. $t_{c}$ is the incapacitation time (in minutes) due to the convective heat and can be calculated from Eq. (5) [15], and the temperature T is obtained from the FDS-based simulation.

$$
t_{c}=\frac{5 \times 10^{3}}{T^{124}}
$$


$t_{y}$ is the incapacitation time (in minutes) due to radiant heat. The calculation formula is shown in Eq. (6) [22], and the radiation flux q can be obtained from the FDS-based simulation.

$$
t_{y}=\frac{1 . a a a}{q^{2.3 a}}
$$

The higher $F_{t}$ is, the more serious the heat hazard. When $F_{t}$ is 1 , smoke heat is fatal.

The mechanisms of smoke toxicity and heat damage are different [14,15], but they are both fatal during enough exposure, therefore the maximum value of both $F_{6 G}$ and $F_{t}$ is the final value of the smoke risk evaluation model, namely SREM, to assess the safety of the evacuation or rescue route in fire. The closer the SREM value is to 1 , the higher the risk is. The route of evacuation or rescue is a specific spatiotemporal curve. The comprehensive risk of the route is determined by the SREM value in the route. And smoke is unevenly distributed and different evacuation or rescue routes have different SREM values. The lower the SREM value is, the more secure the evacuation or rescue path. Therefore, SREM-based VR training can help trainees identify the safest evacuation or rescue path through multiple trainings.

\section{Results and discussion}

In order to verify the validity of the multi-role collaborative virtual fire drill system with smoke risk evaluation, it was tested with real participants. When a participant performs virtual training, he or she logs in to the system by entering a user name and password and selects the version (standalone version and multiplayer version), the game mode (simple, medium, difficult, and custom modes), the role (fireman, police, instructor), And finally into the main scene for virtual training, three trainees client login system simultaneously for collaborative fire drills training, depicted in Fig. 2.

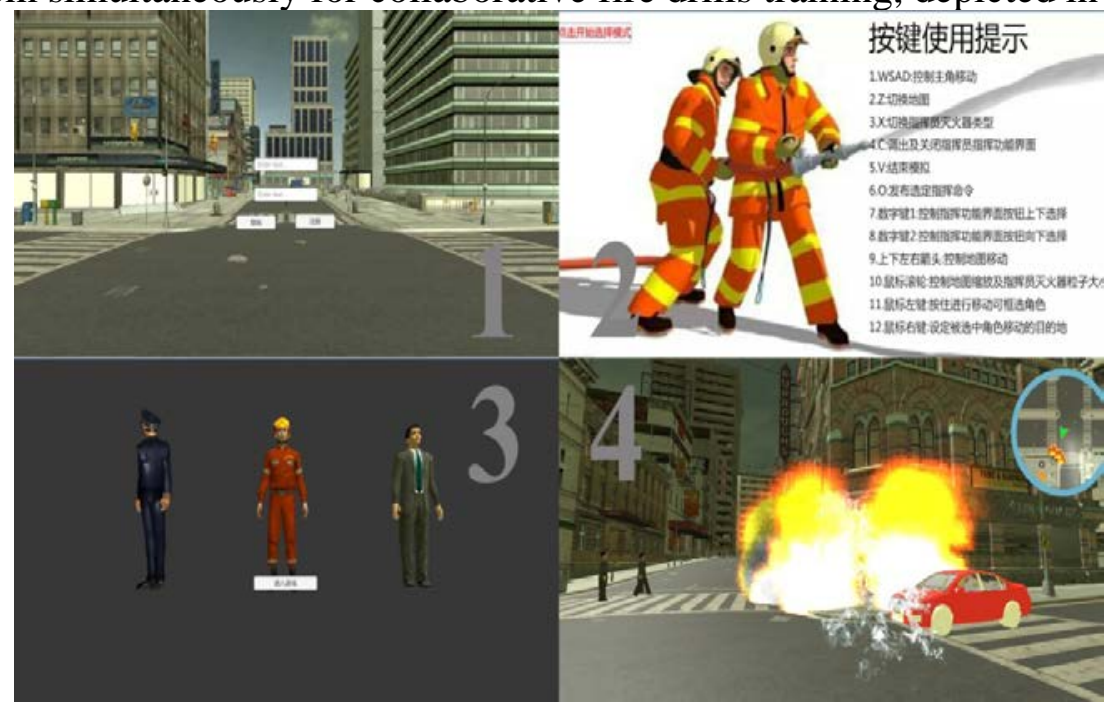

Fig. 2 the result of multi-role collaborative fire drills training

To accurately verify smoke simulation, the correlation coefficients between the distribution of the soot densities by FDS and the distribution of smoke in a virtual scene are calculated by Eq. (7).

$$
\rho \operatorname{ayg}=\frac{\operatorname{cov}(x y)}{\sqrt{\operatorname{Var}(x) \operatorname{Var}(2)}}
$$

The closer the correlation coefficient is to 1, the more similarity between the distribution of smoke in the FDS simulation and the distribution of smoke in the virtual scene is. The change of correlation coefficient in result is always close to 1.0 and the maximum error is only $3.2 \times 10-4$. Thus, it is found that the space-time distribution of smoke in the virtual scene is consistent with that of FDS simulation, which ensures realistic and accurate smoke simulation for training.

The risk of smoke ( i.e. SREM) in path 1 and path 2 (as shown in Fig. 3 ) were calculated for the purpose of accurately verifying the safety of two evacuation routes. In the virtual training, the evacuation speed is approximately from 0.8 to $2.0 \mathrm{~m} / \mathrm{s}$ [6]. The evacuation or rescue time of virtual training in path 1 and path 2 are 26.5s and 21s, respectively. Owing to the visibility limitation of the smoke, the escape speed in path 1 is faster than that in path 2, which results in no obviously different evacuation or rescue time, but the difference of the smoke risk (i.e. SREM) in the two evacuation or 
rescue paths is remarkable. The SREM value in path 2 is 0.35 , while that in path 1 is only 0.06 , nearly only one-sixth of path 2 . If the evacuation or rescue times in path 1 and path 2 is similar, path 1 is safer, so path 1 is a better choice.

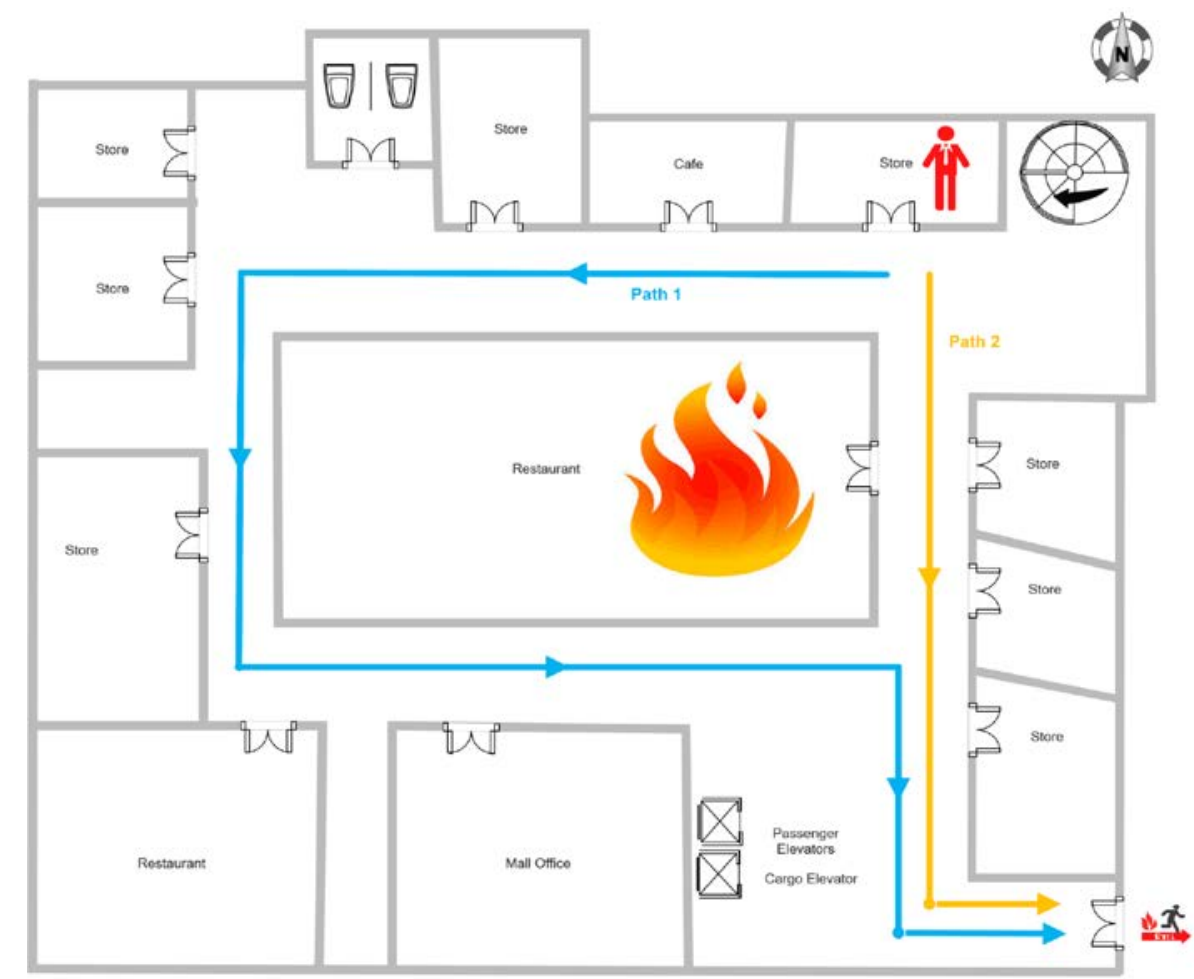

Fig. 3 path 1 and path 2 in virtual fire scene

\section{Conclusion}

The multi-role collaborative fire drill system, supported by the fire dynamics data of FDS and 3D rendering technology, adopts VIZARD as VR platform and KBENGINE as server framework. The participants can joint simulation exercises without constraints on time and space. In order to truly and effectively simulate the smoke in the actual fire scene, FDS data and 3D rendering technology were used. Smoke risk evaluation model was introduced to evaluate the safety of different evacuation or rescue routes, so that trainees can identify the safest escape route. The test results show that the system has good performance and high accuracy, which helps trainees learn the knowledge of firefighting in the low-cost, high-security, high-fidelity and repeatable virtual environment, and accumulate the firefighting actual combat experience.

\section{Acknowledgments}

This work was financially supported by Fujian provincial educational department fund (JAT160389).

\section{References}

[1] Karter MJ. 2011. Fire loss in the United States during 2010. Quincy, MA (USA): NationalFireProtectionAssociationFire.

[2] Flynn JD. 2010. Characteristics of home fire victims. Quincy, MA (USA): National Fire Protection Association.

[3] Lestari F, Green AR, Chattopadhyay G, Hayes AJ. 2006. An alternative method for fire smoke toxicity assessment using human lung cells. Fire Safety J, Vol. 41, No. 605-615.

[4] Cha M, Han S, Lee J, Choi B. 2012. A virtual reality based fire training simulator integrated with fire dynamics data. Fire Safety J, Vol. 50, No. 12-24. 
[5] Ren A, Chen C, Luo Y. 2008. Simulation of emergency evacuation in virtual reality. TsinghuaScience \& Technology, Vol. 13, No. 674-680.

[6] Xu Z, Lu X Z, Guan H, et al. 2014. A virtual reality based fire training simulator with smoke hazard assessment capacity. Advances in Engineering Software, Vol. 68(2), No. 1-8.

[7] Kobes M, Helsloot I, de Vries B, et al. 2010. Way finding during fire evacuation; an analysis of unannounced fire drills in a hotel at night. Build Environ, Vol. 45, No. 537-548.

[8] Chittaro L, Ranon R. 2009. Serious games for training occupants of a building in personal fire safety skills. In: VS-GAMES09 games and virtual worlds for serious applications, March 23-24, 2009, Coventry (UK), No. 76-83.

[9] Chiba N, Muraoka K, Takahashi H, et al. 1994. Two-dimensional visual simulation of flames, smoke and the spread of fire. J Visual Comp Animat, Vol. 5, No. 37-53.

[10]Freund E, Rossmann J, Bucken A. 2005. Fire-training in a virtual-reality environment. In: Proceedings of SPIE-The International Society for Optical Engineering, San Josea (USA), No. 388-394.

[11]Olenick SM, Carpenter DJ. 2003. An updated international survey of computer models for fire and smoke. J Fire Prot Eng, Vol. 13, No. 87-110.

[12] McGrattan K, McDermott R, Hostikka S, Floyd J. 2012. Fire Dynamics Simulator (version 5) Technical Reference Guide. Baltimore, MD (USA): National Institute of Standards and Technology.

[13]Hartzell GE, Priest DN, Switzer WG. 1985. Modeling of toxicological effects of fire gases: II. mathematical modeling of intoxication of rats by carbon monoxide and hydrogen cyanide. $J$ Fire Sci, Vol. 3, No. 115-128.

[14] Irvine DJ, McCluskey JA, Robinson IM. 2000. Fire hazards and some common polymers. Polym Degrad Stabil, Vol. 67, No. 383-396.

[15]Purser DA. 2000. Toxic product yields and hazard assessment for fully enclosed design fires. Polym Int, Vol. 49, No. 1232-1255.

[16]Purser DA. 2002. Toxicity assessment of combustion products. In: The SFPE handbook of fire protection engineering (3rd ed). Quincy, MA (USA): National Fire Protection Association.

[17]British Standards Institution. 1997. Fire safety engineering in buildings, DD 240. London (UK): British Standards Institution.

[18]Babrauskas V, Levin BC, Gann RG, et al. 1992. Toxic potency measurement for fire hazard analysis. Fire Technol, Vol. 28(2), No. 163-7.

[19]Forney GP, Madrzykowski D, McGrattan KB, et al. 2003. Understanding fire and smoke flow through modeling and visualization. IEEE Computer Graphics and Applications, Vol. 23, No. 6-13.

[20]Kaufman A.1990. Volume visualization. Visual Comput, Vol. 6, No. 1-1.

[21]Tang FQ, Ren AZ. 2012. GIS-based 3D evacuation simulation for indoor fire. Build Environ, Vol. 49, No. 193-202.

[22]Purser DA. 1997. Review of Human response to thermal radiation. Contract Research Report No.97/1996. Fire Safety J, Vol. 28, No. 290-291. 